\title{
On the Robustness of Deep Learning Based Beamforming for MU-MISO Systems
}

\author{
Ezequias M. S. de Santana Jr., Igor M. Guerreiro, Yuri C. B. Silva and Charles C. Cavalcante
}

\begin{abstract}
In this paper, we address the problem of robust beamforming in multi-user multiple-input single-output (MUMISO) systems. To this end, we assess the performance of a deep neural network (DNN)-based beamforming strategy under imperfect channel state information (ICSI) conditions in a MUMISO scenario. A robustness analysis is provided by adding a controlled additive error term in the fast fading component of the channel. Such an additive error is independent of the channel realization and does not change the statistics of the channel. The DNN-based beamforming strategy is compared with the classical weighted minimum mean square error (WMMSE) solution in terms of the impact of ICSI on the average sum rate. Numerical results show that ICSI affects in a similar way both the DNNbased strategy and the WMMSE solution. Thus, besides being an alternative method with less computation complexity due to its offline training phase, the DNN-based beamforming strategy can be said to be as robust as the WMMSE solution under ICSI.
\end{abstract}

Keywords-Deep learning; MU-MISO; WMMSE; Beamforming

\section{INTRODUCTION}

On the evolution of wireless communication technologies, it is of paramount importance the optimization of the resources and mechanisms of the network in order to meet the requirements of modern communication systems, providing robustness and better quality of service. Fifth generation $(5 \mathrm{G})$ systems seek to provide reliable connectivity at any time in the most diverse scenarios [1]. 5G system aims to offer low latency, high transmission rates and high mobility, even in denser networks where a high demand for data per user is present, outperforming previous generations and increasing the number of potential applications of mobile systems. The use of robust optimization techniques is essential to provide the proper and efficient functioning of these systems, ensuring that such requirements will be met.

Beamforming (BF) is a means of exploiting antenna arrays to provide the expected performance gains, such as spatial multiplexing to one or multiple users in multi-antenna systems in $5 \mathrm{G}$, by using optimization techniques [2]. One of these techniques is based on the solution for the sum rate maximization problem subject to a total power constraint, which generally results in a non-convex numerical optimization problem.

E. M. S. de Santana Jr., I. M. Guerreiro, Y. C. B. Silva and C. C. Cavalcante are with the Wireless Telecommunications Research Group, Federal University of Ceará, Fortaleza, Brazil, e-mail: \{ezequias, igor, yuri, charles\}@ gtel.ufc.br. This work was supported by Ericsson Research (technical cooperation contract UFC.49), Coordenacão de Aperfeiçoamento de Pessoal de Nivel Superior Brasil (CAPES) under Finance Code 001, by CNPq Proc. 306845/2020-2, by CNPq Proc. 315417/2018-8 and by CNPq Proc. 313151/2020-2.
Suboptimal solutions for that type of problem can be obtained via iterative algorithms such as weighted minimum mean square error (WMMSE) [3], given the optimal solution is usually hard to obtain and impractical for high performance systems that require low latency. However, iterative solutions have some drawbacks related to their computational complexity and delay, especially with the expected increase in the number of antennas in 5G systems. Thus, such classical beamforming techniques may be unable to meet the requirements of real time applications.

Such limitations motivate the search for alternative methods to ensure proper system operation. In this context, machine learning tools have been extensively employed to optimize modern wireless communication systems [4]. For instance, the employment of deep neural networks (DNNs) [5] is suitable to obtain the optimal beamforming in real time due to the possibility of an offline training model. In [6], a framework is proposed for optimal BF considering downlink (DL) transmission, where numerical results show gains with respect to computational complexity with satisfactory performance in a set of common BF problems. However, the work in [6] does not provide any robustness analysis of the proposed DNN under imperfect channel state information (ICSI) conditions, which can severely limit the performance gain of BF techniques [7].

In a recent work [8], another type of machine learning strategy is applied to solve the rate maximization problem in a multi-user multiple-input single-output (MU-MISO) scenario. The authors show that deep Q-learning algorithms can learn a policy that can provide a robust BF structure, improving the performance of models based on perfect channel state information (CSI) in scenarios with ICSI. However, to achieve that goal, the deep Q-learning network needs to be trained from scratch for each execution after being deployed, while in [6] the DNN is designed to be applied directly after an offline training process.

In this paper, we assess the application of a DNN-based beamforming strategy to solve the sum rate maximization problem assuming different levels of CSI imperfection in a MU-MISO system. A robustness analysis of the adopted DNN is provided, showing that it has a similar behavior under ICSI with respect to sum rate degradation when compared to the classical WMMSE solution.

The remainder of this paper is organized as follows. Section II presents the considered system model. Section III is devoted to the beamforming strategies and describes the employed deep learning framework. In Section IV, we present the performance analysis and numerical results. Finally, in Section V, we state the conclusions of this work. 


\section{System Model}

We consider a MU-MISO system in DL where a base station (BS) equipped with an antenna array with $M$ omnidirectional antenna elements serves $K$ user equipments (UEs), each with a single omnidirectional antenna. The channel between the BS and the $k$-th UE is given by

$$
\mathbf{h}_{k}=\sqrt{g_{k}} \tilde{\mathbf{h}}_{k}
$$

where $\tilde{\mathbf{h}}_{k} \in \mathbb{C}^{M \times 1}$ denotes the fast fading component modeled by a complex Gaussian random vector with zero mean and covariance matrix $\mathbf{I}_{M}$, and $g_{k}$ represents the large-scale fading gain.

The channel responses of the UEs are assumed to be known at the BS for beamforming purposes. An ICSI model is adopted by adding noise to the channel fading following the relation [9] [10]:

$$
\hat{\mathbf{h}}_{k}=\sqrt{g_{k}}\left(\sqrt{1-\theta^{2}} \tilde{\mathbf{h}}_{k}+\theta \mathbf{h}_{e}\right)
$$

where $\hat{\mathbf{h}}_{k}$ is the imperfect channel response of the $k$-th UE available at the $\mathrm{BS}$, due to imperfect channel estimation of the direct link, $\mathbf{h}_{e}$ is the independent fast fading error component also modeled by a complex Gaussian random vector with zero mean and covariance matrix $\mathbf{I}_{M}$ uncorrelated with $\tilde{\mathbf{h}}_{k}$, and $0 \leq \theta \leq 1$ represents the accuracy of the channel estimation process. When $\theta=0$ the channel estimate is perfect, whereas when $\theta$ is close to 1 , more uncorrelated is the estimate with the actual channel. The linear combination in (2) ensures that $\mathbf{h}_{k}$ and $\hat{\mathbf{h}}_{k}$ have the same statistics.

For unicast data transmission, the BS applies the beamforming vector $\mathbf{w}_{k} \in \mathbb{C}^{M \times 1}$ to transmit the $M=K$ independent data stream $x_{k}$ to the $k$-th UE. Each data stream is considered to have $\mathbb{E}\left\{\left|x_{k}\right|^{2}\right\}=1$.

The received signal at the $k$-th UE is given as follows:

$$
y_{k}=\mathbf{h}_{k}^{H} \sum_{i=1}^{K} \mathbf{w}_{i} x_{i}+z_{k}
$$

where $z_{k}$ denotes the additive white Gaussian noise with zero mean and variance $\sigma^{2}$. The signal-to-interference-plus-noise ratio (SINR) at receiver $k$ is defined as follows:

$$
\gamma_{k}=\frac{\left|\mathbf{h}_{k}^{H} \mathbf{w}_{k}\right|^{2}}{\sum_{i=1, i \neq k}^{K}\left|\mathbf{h}_{k}^{H} \mathbf{w}_{i}\right|^{2}+\sigma^{2}} .
$$

In this scenario, the beamforming vectors are designed according to the following optimization problem [6]:

$$
\begin{aligned}
& \max _{\mathbf{w}_{1}, \ldots, \mathbf{w}_{K}} \sum_{k=1}^{K} \alpha_{k} \log _{2}\left(1+\gamma_{k}\right) \\
& \text { subject to }\left\|\mathbf{w}_{k}\right\|^{2} \leq P_{\max }, \forall k \in\{1, \ldots, K\} .
\end{aligned}
$$

The goal in (5) is to jointly find the beamforming vectors $\mathbf{w}_{k}$ that maximize the weighted sum rate $\sum_{k=1}^{K} \alpha_{k} \log _{2}(1+$ $\gamma_{k}$ ) subject to a transmit power constraint $P_{\max }$, where $\alpha_{k}$ represents constant system weights for the $k$-th UE.
For the problem (5), as well as in [6], the optimal beamforming vectors can be obtained through the solution structure presented in [11]:

$$
\mathbf{w}_{k}^{\star}=\sqrt{p_{k}} \tilde{\mathbf{w}}_{k}=\sqrt{p_{k}} \frac{\mathbf{h}_{k}\left(\mathbf{I}_{M}+\sum_{j=1}^{K} \frac{\lambda_{j}}{\sigma^{2}} \mathbf{h}_{j}^{H} \mathbf{h}_{j}\right)^{-1}}{\left\|\mathbf{h}_{k}\left(\mathbf{I}_{M}+\sum_{j=1}^{K} \frac{\lambda_{j}}{\sigma^{2}} \mathbf{h}_{j}^{H} \mathbf{h}_{j}\right)^{-1}\right\|} .
$$

According to the solution structure in (6), the optimal beamforming vector for the $k$-th UE is composed of a set of key parameters: a power factor $p_{k}$, a unitary vector that indicates the beamforming direction $\tilde{\mathbf{w}}_{k}$, and the variable $\lambda_{j}$, which represents the Lagrange multipliers for the rate maximization dual problem and obeys the relationship $\sum_{k=1}^{K} p_{k}=$ $\sum_{k=1}^{K} \lambda_{k}=P_{\max }$, as indicated by the strong duality relation discussed in [11].

\section{Multi-User BeAmforming StRategies}

As seen in the previous section, we need to solve the optimization problem (5) to find the precoding vectors that maximize the sum rate of all users. This type of problem is well known in the literature [3], [12], and the process for obtaining an optimal solution is quite complex due to the nonconvex nature of the optimization problem. Next, we present the beamforming strategies considered in this work.

\section{A. WMMSE Beamforming}

Suboptimal solutions to problem (5) can be found via iterative methods such as the WMMSE algorithm described in [3]. To solve the weighted sum rate (WSR) maximization problem for each user rate in the MU-MISO scenario, the WMMSE algorithm will be considered in this work.

As described in [3], the algorithm alternately iterates between the WMMSE transmit filter, the receive filter that minimizes the mean square error (MSE) of the transmit data, and the update of the weight matrix through closed form expressions, exploiting the relationship between the WMMSE and WSR optimization problems to obtain the beamforming vectors.

In the considered scenario, the beamforming matrix $\mathbf{W}=$ $\left[\mathbf{w}_{1}, \ldots, \mathbf{w}_{k}\right]$ is given by [3]:

$$
\mathbf{W}=\left(\mathbf{H}^{H} \mathbf{V}^{H} \mathbf{U V H}+\frac{\operatorname{Tr}\left(\mathbf{U V V} \mathbf{V}^{H}\right)}{P_{\max }} \mathbf{I}_{M}\right)^{-1} \mathbf{H}^{H} \mathbf{V}^{H} \mathbf{U},
$$

where $\mathbf{U}=\operatorname{diag}\left\{u_{1}, \ldots, u_{k}\right\}$ and $\mathbf{V}=\operatorname{diag}\left\{v_{1}, \ldots, v_{k}\right\}$ are the diagonal matrices containing the user WMMSE weights and the MSE filter coefficients, respectively, and $\operatorname{Tr}(\cdot)$ stands for the trace of the argument matrix.

The combination of these weights and filter coefficients will be used as output values in order to train the neural network described in the following subsection.

\section{B. Deep Learning Based Beamforming Framework}

In [6], a multi-user beamforming solution is proposed, which considers the MU-MISO case and employs a machine learning strategy, more precisely, a deep neural network. This strategy allows the learning of suboptimal beamforming 


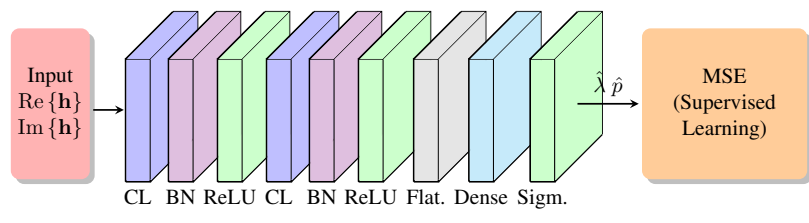

Fig. 1. DNN framework for downlink beamforming.

vectors in real time with a good performance and less computational delay, assuming that offline training models will be used in real time applications.

Based on the sum rate maximization strategy proposed in [6], this work assumes a supervised model that has as input the channel samples between the BS and each UE, and as output values the key parameters $\lambda_{k}$ and $p_{k}$ of the beamforming vectors from (6). Hence, it is possible to recover the beamforming vector using the DNN output and the channel samples.

The output values $\lambda_{k}$ and $p_{k}$ are extracted from the solution given by the WMMSE algorithm presented in (7) to be used in the training of the supervised model. The transmit power values $p_{k}$ can be extracted directly from the columns of the beamforming matrix $\mathbf{W}$ as follows

$$
p_{k}=\left\|\mathbf{w}_{k}\right\|^{2}
$$

whereas the variables $\lambda_{k}$ can be interpreted as user priorities [11] and are given by

$$
\lambda_{k}=P_{\max }\left|\frac{u_{k} v_{k} v_{k}^{*}}{\operatorname{Tr}\left(\mathbf{U V V} \mathbf{V}^{H}\right)}\right|,
$$

where $(\cdot)^{*}$ stands for the complex conjugate operator.

For the construction of the neural network models we consider a DNN structure as illustrated in Fig. 1 which uses supervised learning approach to estimate the output values $\left(\lambda_{k}\right.$, $p_{k}$ ) considering the MSE metric.

Based on [6], the layers that make up the proposed model are listed below.

- Input layer: The complex channel coefficients between the BS and all UEs are stacked into a channel data vector, e.g. $\mathbf{h}=\left[\mathbf{h}_{1}^{T}, \ldots, \mathbf{h}_{K}^{T}\right]^{T} \in \mathbb{C}^{M K \times 1}$, and then separated into real and imaginary components before they are fed in batches into the neural network. Then, the input data of the neural network are in the form $[\operatorname{Re}\{\mathbf{h}\}, \operatorname{Im}\{\mathbf{h}\}]^{T} \in$ $\mathbb{R}^{2 \times M K}$.

- Convolutional Layers: In each convolutional layer the input layer is convolved with the convolutional kernels, and their parameters, such as weights and bias, are shared among different channel coefficients to extract the features.

- Batch Normalization layers: The normalization layers are introduced after each convolutional layer to normalize their output by subtracting the batch mean and dividing by the batch standard deviation. As described in [6], these layers help to reduce the probability of over-fitting, to enable a higher learning rate, and to make the neural network less sensitive to the initialization of weights.
- Activation (Ac.) layers: These layers are introduced after the batch normalization layers to apply the rectified linear unit (ReLU) or sigmoid function to the input.

- Flatten Layer and Fully-connected Layer: The former is used to reshape its input to a vector that will be interpreted by the fully-connected layer, that applies the weights and the biases from its neurons to its input.

- Output layer: Here the predicted key parameters vector $\left[\hat{\mathbf{p}}^{T}, \hat{\lambda}^{T}\right]^{T} \in \mathbb{R}^{2 K \times 1}$ are generated, where $\mathbf{p}$ and $\lambda$ are the vectors containing the power and the weights of all users respectively, and the MSE loss function is applied finishing the learning process.

After applying the trained model to the channel samples, the predicted output values are converted to suboptimal key parameters by a scaling process to meet the power constraint using the following expressions:

$$
\hat{\mathbf{p}}^{\star}=\frac{P_{\max }}{\|\hat{\mathbf{p}}\|_{1}} \hat{\mathbf{p}} \text { and } \hat{\lambda}^{\star}=\frac{P_{\max }}{\|\hat{\lambda}\|_{1}} \hat{\lambda}
$$

With the scaled parameters, the optimum solution structure (6) is applied to recover the estimated optimum beamforming vector $\mathbf{w}_{k}^{\star}$ considering the values of $\hat{\lambda}^{\star}$ and $\hat{\mathbf{p}}^{\star}$ defined in (10).

TABLE I

NeUral Network LAyer Parameters.

\begin{tabular}{ll}
\hline \# Layer & Description \\
\hline 1. Input Layer & Size $=2 \times M K ; 200$ batches; 100 epochs \\
2. Conv. Layer (CL) & $8(3 \times 3)$ kernels with padding $(1,1) ;$ stride 1 \\
3. Batch Norm. Layer (BN) & Momentum $=0.99 ; \varepsilon=0.001$ \\
4. Ac. Layer & ReLU \\
5. Conv. Layer (CL) & $8(3 \times 3)$ kernels with zero padding 1; stride 1 \\
6. Batch Norm. Layer (BN) & Momentun $=0.99 ; \varepsilon=0.001$ \\
7. Ac. Layer & ReLU \\
8. Flatten Layer & \\
9. Fully-connected Layer & Dense with $2 K$ neurons \\
10. Ac. Layer & Sigmoid \\
11. Output Layer & MSE metric; Adam optimizer with learning rate of 0.001 \\
\hline
\end{tabular}

\section{Simulation Results}

In order to assess the performance of the BF strategies and evaluate their robustness considering ICSI, we consider the DL transmission system described in Section II composed of a single BS equipped with $M=4$ antennas that serves $K=$ 4 single antenna UEs. The large scale fading component $g_{k}$ follows the model $g_{k}=128.1+37.6 \log _{10}\left(r_{k}\right)[\mathrm{dB}]$ [13] where $r_{k}$ is the distance in $\mathrm{km}$ between the BS and the $k$-th UE. UE positions are generated uniformly inside a circle of radius $500 \mathrm{~m}$ and centered at the BS position, considering a minimum distance of $100 \mathrm{~m}$ from the $\mathrm{BS}$, the considered noise power spectral density is $-174 \mathrm{dBm} / \mathrm{Hz}$, and the system bandwidth is $20 \mathrm{MHz}$. The simulation parameters are summarized in Table II.

For the training of the DNN models, a set of 25,000 samples $(\mathbf{h},[\lambda, p])$ were considered, with a train-test split ratio of $80 \% / 20 \%$. As in [6], the weights of the network layers were initialized following a Glorot normal distribution and the biases initially set to 0 . Also, data normalization is applied by scaling the channel samples by the noise power. The targets were divided by a factor to ensure that they are contained 
TABLE II

Simulation PARAMETERS.

\begin{tabular}{lc}
\hline Number of BS antennas $M$ & 4 \\
Number of single antenna UEs $K$ & 4 \\
Cell radius & $500 \mathrm{~m}$ \\
UE-BS min. distance & $100 \mathrm{~m}$ \\
System bandwidth & $20 \mathrm{MHz}$ \\
Noise power spectral density & $-174 \mathrm{dBm} / \mathrm{Hz}$ \\
\hline
\end{tabular}

within the interval $[0,1)$ to guarantee numerical stability. The other parameters of the neural network are described in Table I. The DNN models and the benchmarks were implemented using Python 3.8 with Tensorflow 2.4, Keras 2.4.3 and Numpy 1.20 as numerical computing library on a computer with 1 AMD Ryzen5-3600X CPU Core and RAM of 32GB.

To compare the performance of the BF strategies as solution for the optimization problem (5), the zero forcing (ZF) and regularized zero forcing (RZF) [11] solutions with equal power allocation were considered as well as the WMMSE beamforming with the initialization based on RZF and the system weights $\alpha_{k}=1$ as baseline solutions for comparing with the DNN beamforming strategy. Also, the target key parameters were extracted from the WMMSE solution to compose the training and test sets.

All the results were generated considering the average of 5, 000 experiments. In Fig. 2 it is observed that with the increase in the transmitted power there is an increase in the sum rate performance, with the DNN solution showing itself as an upper limit for the RZF solution and close to the WMMSE target for low signal-to-noise ratio (SNR). In this case, the supervised learning solution tries to approach the WMMSE solution as much as possible, but its performance is upper limited by the WMMSE and lower bounded by the WMMSE initialization, showing the potential of the DNN Beamforming.

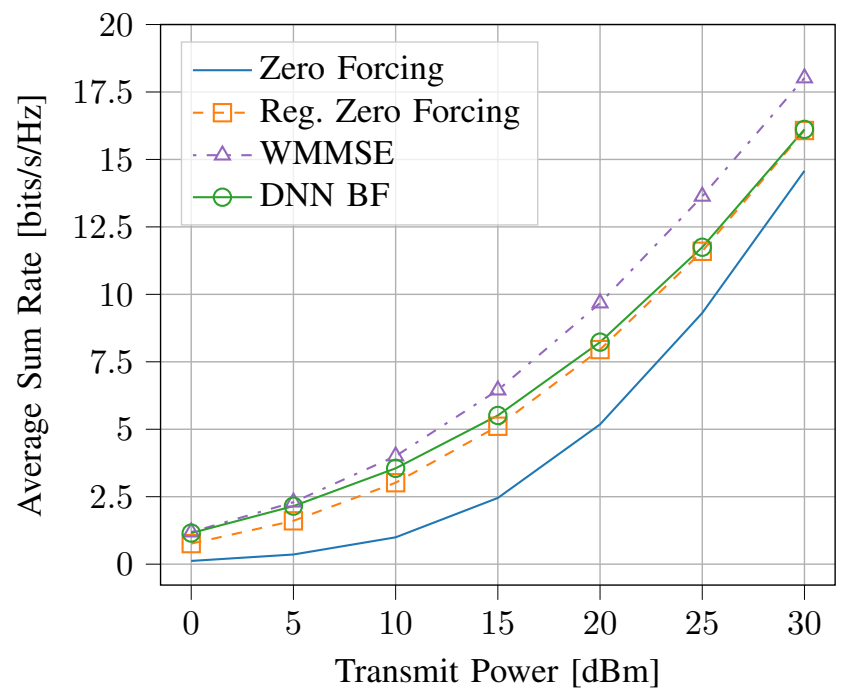

Fig. 2. Sum rate performance comparison considering a system with $M=K=4$.

In Fig. 3, we assess the execution time of the BF strategies. As the number of transmit antennas increases, the DNN BF applied after an offline training process have a reduced compu- tational complexity in comparison with the iterative WMMSE solution. The ZF and RZF presented the best execution times due to their vectorized implementation. The main source of complexity in the DNN BF computations is the recovery process using (6).

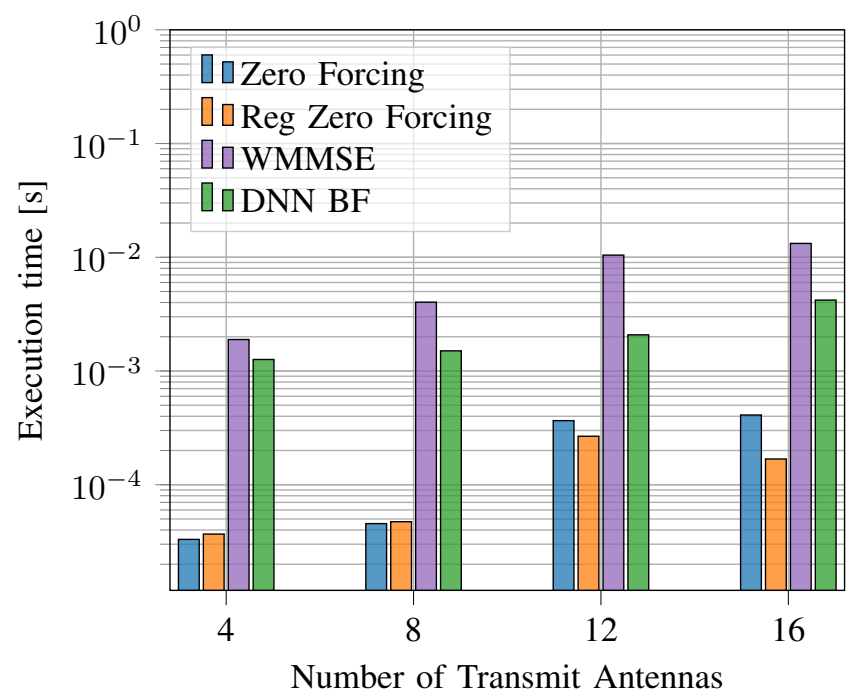

Fig. 3. Execution time per sample considering a system with $P_{\max }=30 \mathrm{dBm}$ and $M=K$.

For the robustnesss analysis of the supervised learning solution, we consider the ICSI model (2), with the WMMSE performance as a benchmark. First, we perform the experiments applying the WMMSE solution obtained using $\hat{\mathbf{h}}_{k}$, varying the parameter $\theta$ between $[0,1]$, and compute the error between the average achieved sum rate and the sum rate that would be achieved when considering perfect CSI.

To investigate the performance of the DNN beamforming, we consider $\hat{\mathbf{h}}_{k}$ as the input model for the precoder prediction. Then, we compute the average sum rate and the error considering the predicted precoder using perfect CSI samples.

The relative error (\%) results for both the WMMSE and DNN beamforming are summarized in tables III and IV. We see that the relative error obtained using the DNN solution increases as much as the error obtained using WMMSE with the evolution of the transmit power. This behavior indicates that the channel estimation error, which follows the same statistical distribution of the channel coefficients, has a similar effect on both solutions.

TABLE III

Avg. Sum Rate Error due to ICSI for Each $P_{\text {Max }}-$ WMMSE BF

\begin{tabular}{lrrrrrrr}
\hline & $0 \mathrm{dBm}$ & $5 \mathrm{dBm}$ & $10 \mathrm{dBm}$ & $15 \mathrm{dBm}$ & $20 \mathrm{dBm}$ & $25 \mathrm{dBm}$ & $30 \mathrm{dBm}$ \\
\hline$\theta=0$ & 0.00 & 0.00 & 0.00 & 0.00 & 0.00 & 0.00 & 0.00 \\
$\theta=0.25$ & 3.85 & 4.12 & 5.86 & 9.66 & 15.83 & 25.31 & 37.51 \\
$\theta=0.5$ & 15.19 & 16.05 & 20.65 & 29.14 & 40.13 & 52.38 & 63.99 \\
$\theta=0.75$ & 33.56 & 35.48 & 41.59 & 51.42 & 61.89 & 71.53 & 79.16 \\
$\theta=1$ & 61.86 & 62.89 & 67.96 & 75.17 & 81.31 & 85.91 & 89.28 \\
\hline
\end{tabular}

For each value of $\theta$, we can see in Fig. 4 the sum rate performance of the beamforming solutions. The DNN based precoder performance is close to that of WMMSE for low 
TABLE IV

AVG. Sum RATE ERror DUE TO ICSI FOR EACH $P_{\text {MAX }}-$ DNN BF

\begin{tabular}{lrrrrrrr}
\hline & $0 \mathrm{dBm}$ & $5 \mathrm{dBm}$ & $10 \mathrm{dBm}$ & $15 \mathrm{dBm}$ & $20 \mathrm{dBm}$ & $25 \mathrm{dBm}$ & $30 \mathrm{dBm}$ \\
\hline$\theta=0$ & 0.00 & 0.00 & 0.00 & 0.00 & 0.00 & 0.00 & 0.00 \\
$\theta=0.25$ & 3.58 & 3.51 & 4.97 & 7.98 & 13.46 & 23.45 & 37.55 \\
$\theta=0.5$ & 14.40 & 14.33 & 18.59 & 26.31 & 37.09 & 51.01 & 64.42 \\
$\theta=0.75$ & 32.82 & 32.43 & 39.04 & 49.03 & 59.66 & 70.58 & 79.30 \\
$\theta=1$ & 60.82 & 60.45 & 66.83 & 74.20 & 79.84 & 84.70 & 88.58 \\
\hline
\end{tabular}

SNRs as in Fig. 2, and the sum rate curve behavior in the presence of channel estimation error shows that with the increase of $\theta$, both strategies are similarly penalized, with the WMMSE performing better than the supervised solution as expected.

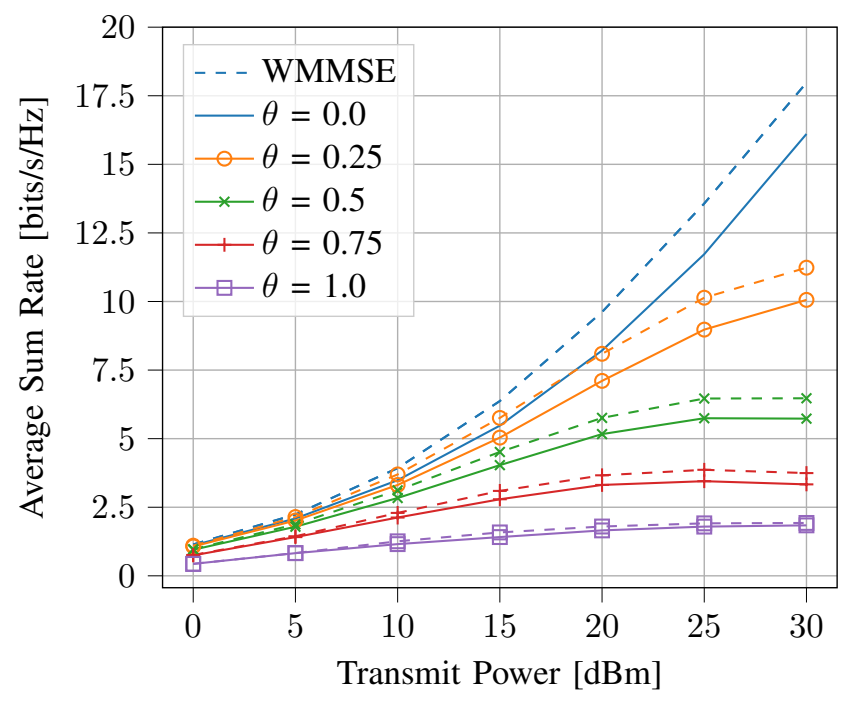

Fig. 4. Sum rate performance for WMMSE (dashed patterns) and DNN BFs (solid) in presence of different levels of ICSI controlled by the variable $\theta$.

\section{CONCLUSION}

In this work we asses the performance of a DNN BF solution for a MU-MISO system under ICSI. The ICSI was modeled as a combination of the actual channel with a controlled noisy term. The focus was on the maximization of the weighted sum rate, which is by definition a non-convex problem whose parameterized optimal solution is hard to be obtained. The DNN BF applied to obtain a suboptimal solution is drawn from a DNN whose input is the channel response and output is the beamforming vector parameters, while the (noisy) channel is assumed to be known at the BS.

The results indicate that the deep learning based beamforming solution is viable and as robust as the WMMSE, assuming that the channel and ICSI models follow the same distribution. As perspectives for future works we can consider other ICSI models with different statistical distributions; employ DNN beamforming in a system considering multi-antenna UEs with multiple streams; consider other learning approaches that can take use of the sum rate information as a reward, such as in reinforcement learning.

\section{REFERENCES}

[1] A. Osseiran, S. Parkvall, P. Persson, A. Zaidi, S. Magnusson, and K. Balachandran, "5G wireless access: An overview," Ericsson, White Paper 1/28423-FGB1010937, Apr. 2020, [Online]. Available: https://www.ericsson.com/en/reports-and-papers/white-papers/5gwireless-access-an-overview.

[2] P. von Butovitsch, D. Astely, A. Furuskär, B. Göransson, B. Hogan, J. Karlsson, and E. Larsson, "Advanced antenna systems for 5G networks," Ericsson, White Paper GFMC-18:000530, Nov. 2018, [Online]. Available: https://www.ericsson.com/en/reports-andpapers/white-papers/advanced-antenna-systems-for-5g-networks.

[3] S. S. Christensen, R. Agarwal, E. De Carvalho, and J. M. Cioffi, "Weighted sum-rate maximization using weighted MMSE for MIMOBC beamforming design," IEEE Transactions on Wireless Communications, vol. 7, no. 12, pp. 4792-4799, Dec. 2008.

[4] F.-L. Luo, Machine Learning for Future Wireless Communications, 1st ed. Wiley-IEEE Press, 2020. DOI: $10.1002 / 9781119562306$.

[5] H. Sun, X. Chen, Q. Shi, M. Hong, X. Fu, and N. D. Sidiropoulos, "Learning to optimize: Training deep neural networks for interference management," IEEE Transactions on Signal Processing, vol. 66, no. 20, pp. 5438-5453, Oct. 2018. DOI: $10.1109 /$ tsp. 2018 . 2866382

[6] W. Xia, G. Zheng, Y. Zhu, J. Zhang, J. Wang, and A. P. Petropulu, "A deep learning framework for optimization of MISO downlink beamforming," IEEE Transactions on Communications, vol. 68, no. 3 , pp. 1866-1880, Mar. 2020. DOI: 10 . 1109 / TCOMM . 2019. 2960361.

[7] G. C. Alexandropoulos, P. Ferrand, and C. B. Papadias, "On the robustness of coordinated beamforming to uncoordinated interference and CSI uncertainty," in 2017 IEEE Wireless Communications and Networking Conference (WCNC), May 2017, pp. 1-6. DOI: doi : $10.1109 / \mathrm{WCNC} .2017 .7925853$.

[8] I. M. Braga, E. d. O. Cavalcante, G. Fodor, Y. C. B. Silva, C. F. M. e Silva, and W. C. Freitas, "User scheduling based on multi-agent deep Q-learning for robust beamforming in multicell MISO systems," IEEE Communications Letters, vol. 24, no. 12, pp. 2809-2813, Dec. 2020.

[9] F. Rusek, D. Persson, B. K. Lau, E. G. Larsson, T. L. Marzetta, O. Edfors, and F. Tufvesson, "Scaling up MIMO: Opportunities and challenges with very large arrays," IEEE Signal Processing Magazine, vol. 30, no. 1, pp. 40-60, Jan. 2013.

[10] D. Mi, M. Dianati, L. Zhang, S. Muhaidat, and R. Tafazolli, "Massive MIMO performance with imperfect channel reciprocity and channel estimation error," IEEE Transactions on Communications, vol. 65, no. 9, pp. 3734-3749, Sep. 2017.

[11] E. Björnson, M. Bengtsson, and B. Ottersten, "Optimal multiuser transmit beamforming: A difficult problem with a simple solution structure [lecture notes]," IEEE Signal Processing Magazine, vol. 31, no. 4, pp. 142-148, Jul. 2014.

[12] Q. Shi, M. Razaviyayn, Z. Luo, and C. He, "An iteratively weighted MMSE approach to distributed sum-utility maximization for a MIMO interfering broadcast channel," IEEE Transactions on Signal Processing, vol. 59, no. 9, pp. 4331-4340, Sep. 2011.

[13] 3GPP, TR 36.942: Evolved universal terrestrial radio access (EUTRA); radio frequency $(R F)$ system scenarios, Release 16, Technical report, 2020. 